\title{
COMMISSION 10: SOLAR ACTIVITY
}

\section{(ACTIVITE SOLAIRE)}

\author{
PRESIDENT: G. Ai \\ VICE-PRESIDENT: A. Benz \\ ORGANIZING COMMITTEE: K. P. Dere, O. Engvold, N. Gopalswamy, \\ R. Hammer, A. Hood, B. V. Jackson, I. Kim, P. C. Marten, G. Poletto, \\ J. P. Rozelot, A. J. Sanchez, K. Shibata \& L. van Driel-Geztelyi
}

\section{INTRODUCTION}

The Sun's activity has been evolving in the ascending phase of Solar Cycle 23 since 1996. Similarly, the research on solar activity is also in the ascending phase of a new active period. Numerous new results have been obtained from a large amount of space and ground observations covering a wide spectral range. In particular, observations with YOHKOH, SOHO, and TRACE have revealed a multitude of phenomena and processes in the solar atmosphere which provide us a new picture of the Sun.

It is obvious that all of the progress in research on solar activity can hardly be included in the current report, with limited space. We, therefore, focus on the main scientific developments in the field of Commission 10 during the triennium 1997-1999.

\section{SCIENTIFIC HIGHLIGHTS 1997-1999}

First of all, it should be emphasized that a large amount of new data obtained with spacebased instruments enhance our understanding of the complex phenomena and processes in the solar atmosphere. We find it practical for our overview to divide solar activity into the following three categories: small-scale, medium-scale and large-scale activity.

\subsection{Small-Scale Solar Activity}

Small-scale solar activities listed in this subsection have been observed in solar quiet regions, which demonstrate that the quiet Sun is never 'quiet'. It is believed that these small-scale activities are non-thermal heating processes in the solar atmosphere.

Benz, Krucker and their colleagues reported heating events in quiet regions, and found that these events have many similarity with regular flares, but have less energy than about $10^{27}$ ergs. They have discovered these events in soft X-rays first (Krucker et al., 1997) with YOHKOH, than also in coronal Fe-lines in EIT EUV observations (Benz and Krucker, 1998). Recently, Benz and Krucker (1999) found that the coronal energy input is well correlated to transition region line brightening and to radio enhancements. However, the energy distribution of heating events follows a power law whose exponent is still ill-defined. Depending on whether it is lower, or higher, than -2 , small events may be able, or unable, to provide for coronal heating. Power law indices from observations of different events range from -1.4 (Berghmans et al., 1998) up to -2.6 (Krucker and Benz, 1998), showing that a general consensus on this issue has not yet been reached.

Gopalswamy, Zhang et al., (1997) detected transient microwave brightenings from the peripheries of sunspots using VLA microwave observations which correspond to an energy release rate of only $1.3 \times 10^{22} \mathrm{erg} \mathrm{s}^{-1}$. These observations suggest that thermal energy contents could be as low as $10^{24} \mathrm{erg}$. Koutchmy et al., have found many very faint soft 
$\mathrm{X}$-ray brightenings in polar coronal holes with YOHKOH/SXT, which they called SXR coronal flashes. The energy of each brightening is about $10^{24} \mathrm{erg}$, corresponding to that of nano-flares. Tiny jets are often associated with these brightenings.

Schrijver et al., (1998) pointed out that large-scale coronal heating is closely related to continuous emergence of small bipolar regions on the quiet Sun. Based upon SOHO observations, scientists have introduced the concept of 'magnetic carpet' to illustrate and explain how magnetic energy provides heating of the corona.

Mini-filament eruptions in solar quiet regions have been observed recently. It has been found that the total mass and kinetic energy involved in miniature filament eruptions is about $10^{13} \mathrm{~g}$ and $10^{25} \mathrm{erg}$, respectively (Wang et al., 1998).

TRACE images revealed the presence of a new small-scale structure, the moss, consisting of a highly dynamic bright pattern with dark inclusions, possibly related to the lower sections of hot coronal loops (Berger et al., 1999).

\subsection{Medium-Scale Solar Activity}

Medium-scale solar activity comprise a variety of solar phenomena, such as sunspots, filaments and prominences, regular flares, jets, transient brightenings and so on. With their energy content on medium spatial scale, these phenomena have been observed by various means for a long time, but important questions still remain unresolved. There are hundreds of papers concerning these phenomena published in scientific journals each year. Since space is limited, we present only a few important results in this subsection.

With recent advances in techniques of sunspot observations, a coherent picture of magnetic fields and velocity fields in sunspots is drawn step by step (Plaza et al., 1997; Montesinos and Thomas, 1997; Schlichenmaier et al., 1998, Lites et al., 1998).

As an active field of solar physics, studies of filaments and prominences in recent years focus mainly on plasma properties, fine structures, dynamics and formation (Gaizauskas et al., 1997; Zirker et al., 1998; Kucera et al., 1999). It is being realized that magnetic orientation of filament channels, the barbed structures of quiescent filaments, and the helicity of erupting filaments are intrinsically related to the solar global magnetic patterns of chirality (Zirker et al., 1997). The relation between eruptive prominences and coronal mass ejections (CMEs) have been studied by many. A meeting held in Aussois, France (IAU Colloquium 167) discussed new perspectives on solar prominences.

Solar flares, the traditional subject in the study of solar activity, is still an attractive field of research. A most exciting discovery with SOHO (Kosovichev, 1998) is that solar flares are found to produce seismic waves and gigantic seismic quakes in the Sun's interior. It is widely accepted that a large energy reservoir exists in solar magnetic fields and that magnetic reconnection is probably the energy release process, while the reason for its sudden release is still debated. However, it is apparent from recent YOHKOH, SOHO and TRACE observations (Shibata, 1996; Tsuneta and Naito, 1998; Innes et al., 1997, Thomas, 1998, Schmieder et al, 1997; Démoulin et al; Wang, Wang and Qiu, 1999) and theoretical breakthroughs (Karpen et al., 1996; Priest and Titov, 1996; Galsgaard and Nordlund, 1997; Yokoyama and Shibata, 1998; Chen et al., 1999) that studies of magnetic reconnection are another exciting area of research.

$\mathrm{X}$-ray jets have been observed for a long time. Shimojo et al. (1998) found that more than 70 percent of X-ray jets occurred at mixed magnetic polarities. This is one important line of evidence that $\mathrm{X}$-ray jets are produced by magnetic reconnection. Jets and transient brightenings were recently observed in the radio spectral range. Kundu et al. (1998) detected no type III bursts in association with two-side-loop type jets. Nindos et al. (1999) searched for radio-selected transient brightenings as a complement to more common X-rayselected surveys. Observational data favor an interpretation in terms of gyro-synchrotron radiation from mildly relativistic electrons. Two of the microwave transient brightenings also showed evidence for type III radio emission at $327 \mathrm{MHZ}$. Moreover, EIT observations have revealed similar jets that are occasionally ejected from polar coronal holes (Moses et al., 1997; Wang et al., 1998). 


\subsection{Large-Scale Solar Activity}

We put types of solar activity with long temporal scale and/or large spatial scale into a separate subsection. In that sense, solar cycle, solar radiation variability, large-scale evolving structures, coronal mass ejection and solar wind are regarded as large-scale solar activities. Since some of these contents also appear in the reports from Commissions 12 and 49 , we report here only a few important results related to solar activity.

Long-lived giant cells and a subsurface flow of material from the Sun's equator to its poles have been detected with the SOI/MDI instrument on board the SOHO satellite (Beck et al., 1998; Giles et al., 1997). Both of these observational results might be closely related to solar activity.

The radiation from the Sun is an important factor that determines the Earth's radiation balance and climate system. Solar radiation variability is mainly attributed to solar magnetic phenomena, such as sunspots, faculae, plages, and network, which modify the Sun's net radiative output by altering temperature and density in the otherwise homogeneous solar atmosphere (Lean, 1997). The SOLERS22 International Workshop held at National Solar Observatory/Sacramento Peak during June 17-21, 1996 exhibited new results of solar irradiance measurements in a wide spectral range with instruments on board a series of satellites. Lockwood et al. (1999) recently discovered that the total magnetic flux leaving the Sun has risen by a factor of 1.4 since 1964, and the increase since 1901 has been by a factor of 2.3. This increase has resulted in changes in heliospheric magnetic field, and then those in the Earth's climate (Svensmark and Friis-Christensen, 1997).

Magnetic helicity observed at the solar surface, in the solar corona, and in the solar wind that streams out past Earth, contains unique and useful signatures of the physical conditions and processes deep below the photospheric surface. Solar global magnetic patterns of chirality are intrinsically related to the distribution of magnetic helicity in the solar atmosphere (Low, 1996). Both X-ray images of the solar corona from YOHKOH and measurements of vector magnetic fields in the solar photosphere from ground-based observatories (Canfield et al., 1993; Lites et al., 1994; Pevtsov, 1995; Bao and Zhang, 1998) motivate scientists to improve their understanding of the topology of magnetic fields and plasma flows in the Sun from the deep interior to the solar wind.

CMEs are another important type of solar activity. The launch of SOHO in 1995 has provided a platform for the first nearly continuous observations of the corona since the demise of SMM and P78-1. The combination of Extreme-ultraviolet Imaging Telescope (EIT) and Large Angle Spectrometric Coronagraph (LASCO) on SOHO have allowed the first observations of CMEs including their initiation and their traversal through $30 R_{\odot}$. Dere et al. (1997) reported the first observations of such a CME which started in a small 35 arc-sec. long segment of prominence material. EIT observations have led to the discovery of coronal Moreton waves which are produced at the initiation of some flares and coronal mass ejections (Thompson et al., 1999). It is much easier to detect these waves than the traditional H-alpha Moreton waves. These waves seem to be an early signature of solar eruptive events and have become an important source of information on Earth-directed CMEs.

Other signatures of CMEs in the lower corona include a sudden depletion or intensity 'dimming' of the X-ray corona which sometimes accompany a CME. Sterling and Hudson (1997) identified a dimming that occurred just prior to a 'halo' CME, observed on 1997 April 7 using the Soft X-ray Telescope on YOHKOH. Zarro et al. (1999) reported that coronal dimming at extreme-ultraviolet wavelengths corresponding to the X-ray dimming were observed with the EIT. These observations are consistent with the source of the CME being a flux rope that erupted resulting in a loss of emitting plasma. Gopalswamy and Hanaoka (1998) studied a giant prominence eruption in microwaves preceded by X-ray coronal dimming. Instead of dimming, sometimes a global enhancement is seen in X-rays which seems to be the X-ray counterpart of the CME (Gopalswamy et al., 1999).

Images of CMEs seen in LASCO suggest that they are magnetic flux ropes (Chen et al., 1997; Dere et al., 1999) as found in magnetic clouds. The ejection of flux rope CMEs from 
streamers was proposed by Low (1996) but Submaranian et al. (1999) have found that many CMEs associated with streamers do not appear to have any effect on the streamer. Canfield et al. (1999) find that active regions with X-ray signatures of a sigmoidal morphology are likely to produce eruptive activity.

The geophysical impact of mass ejection has also been of great current interest. Brueckner et al. (1998) found that all but two geomagnetic storms with $\mathrm{Kp} \geq 6$ during March 1996 and June 1997 were caused by 'halo' and partial 'halo' CMEs observed by LASCO. The storms occured roughly 80 hours after the CME was first detected.

Solar wind is the main research field of IAU Commission 49, and progress in this field are presented in the report from that commission.

Finally, it should be pointed out that advances in modern solar instrumentation have led to significant progress in solar physics.

During this triennium, we had a series of space missions for solar observations (see previous reports from IAU Division II), and a number of ground-based instruments which have been put into use, such as THEMIS (Rayrole et al., 1998), PSPT (Ermolli et al., 1998), MCST (Deng et al., 1997). Moreover, in order to support observations of SOHO and TRACE, a number of existing ground-based facilities have been successfully upgraded. For example, a new digital magnetograph system has been installed and tested at Big Bear Solar Observatory (Wang et al., 1998).

The Nobeyama radioheliograph in Japan has become an important tool in studying large scale prominence eruptions and arcade formations in relation to CMEs (Gopalswamy et al, 1997; Hanaoka and Shinkawa, 1999). This microwave array obtains uninterrupted observations for eight hours every day and has become a versatile tool to study solar eruptive events. Metric imaging by the Nancay radioheliograph has helped to better understand white light CME events because it can image structures close to the surface (Pick, 1999). In addition, a broadband radiospectrometer has been set up and preliminary results obtained (Fu et al., 1998 ).

\section{SPECIAL PROJECTS/OTHER ACTIVITIES}

There have been several successful campaigns, where ground-based observatories have provided coordinated support to SOHO, YOHKOH and TRACE. Most of these campaigns were introduced in the websites listed in Section 6

\section{MAJOR SCIENTIFIC CONFERENCES 1997-1999}

"Observational Plasma Astrophysics: Five Years of Yohkoh and Beyond" T. Watanabe, T. Kosugi, A.C.Sterling (eds), Kluwer Academic Pub., 1997

"New Perspectives on Solar Prominences", IAU Coll 167, Aussois, France, 28 April 4 May 1997. ASP Conf. Series 150

"Second Advances in Solar Physics Euroconferences: Three-Dimensional Structure of Solar Active Regions" held in Preveza, Greece 7-11 October 1997. ASP Conf. Series 155

"Solar Jets and Coronal Plumes", Guadeloupe, France, 23-26 February 1998. ESA SP-421. "Solar and Stellar Activity: Similarities and Differences", held in Armagh, N. Ireland 2-4 September 1998. ASP Conf. Series 158

"IAUU Coll. 179 Cyclical Evolution of Solar Magnetic Fields: Advances in Theory and Observations", Kodaikanal, India, Dec. 1999

$I X^{\text {th }}$ European Conference on Solar Physics "Magnetic Fields and Solar Processes", Firenze, Italy, Sept 12-18, 1999

\section{MAJOR PUBLICATIONS 1997-1999}

"The Many Faces of the Sun: A Summary of the Results from NASA's Solar Maximum Mission", Strong, K.T., Haisch, B.M., Schmelz, J.T. Eds., Springer, 1999

"The First Results from SOHO" in 1997 Solar Physics 170 and 175

Proceedings of the Workshop on "Measurements and Analyses of the 3-D Solar Magnetic 
Fields" in 1997 Solar Physics 174

Proceedings of the SOLERS22 International Workshop in 1998 Solar Physics 177

\section{SOURCES OF FURTHER INFORMATION}

(1) http://soho.www.nascom.nasa.gov (2) http://soho.www.estec.esa.nl/

(3) http://www.Imsal.com/TRACE/gateway.html (4) http://www.solar.isas.ac.jp/

(5) http://www.gong.noao.edu/SolarNews/ (6) http://www.iau.org/commissions.html

Guoxiang Ai

President of the Commission

\section{References}

Bao, S. D. and Zhang, H. Q.: 1998, Astrophys. J. 496, L43

Beck, J. G., Duvall Jr, T. L. and Scherrer, P. H.: 1998, Nature, 394, 553

Benz, A. O. and Krucker, S.: 1998, Solar Phys., 182, 349

Benz, A. O. and Krucker, S.: 1999, Astron. Astrophys., 341, 286

Berger, T.E., De Pontieu, B., Schrijver, C. J., Title, A.M.: 1999, Astrophys. J., 519, L97

Berghmans, D., Clette, F., Moses, D.: 1998, Astron. Astrophys., 336, 1039

Brueckner, G. E., Delaboudinere, J.-P., Howard, R. A. et al.: 1998, Geophys. Res. Lett., $25,3019$.

Canfield, R. C., de La Beaujardiere, J.F., Fan, Y. et al.: 1993, Astrophys. J., 411,362

Canfield, R. C., Hudson, H. S., Mckenzie, D. E.: 1999, Geophys. Res. Lett., 26, 627.

Chen, J., Howard, R. A., Brueckner, G. E. et al.: 1997, Astrophys. J., 490, L191.

Chen, P. F., Fang, C., Tang, Y. and Ding, M. D.: 1999, Astrophys. J., 513, 516

Démoulin, P., Bagala, L. G., Mandrini, C. H. et al.: 1997, Astron. Astrophys., 325, 305

Deng, Y. et al.: 1997, Solar Phys., 173, 207

Dere, K P., Brueckner, G. E., Howard, R. A. et al.: 1997, Solar Phys., 175, 601.

Dere, K. P., Brueckner, G. E., Howard, R. A. et al.: 1999, Astrophys. J., 516, 465.

Ermolli, Fofi, M., Bernacchia, C., Berrilli, F., Caccin, B., Egidi, A. and Florio, A.: 1998, Solar Phys., 177, 1

Fu, Q. J., Liu, Y. Y., Ji, H. R. and Huang, G. L.: 1998, Nobeyama Symposium on Solar Physics with Radio Observations, Oct. 27-30, 1998, Kiyosato, Japan, p19

Gaizauskas, V., Zirker, B. J., Sweetland, C., and Kovacs, A.: 1997, Astrophys. J., 479, 448

Galsgard, K. and Nordlund, A. J.: 1997, Geophys. Res., 102, 231

Gopalswamy, N. and Hanaoka, Y.: 1998, Astrophys. J., 498, L179, 1998

Gopalswamy, N., Zhang, J., Kundu, M. R., Schmahl, E.J., and Lemen, J. R.: 1997, Astrophys. J., 491, L115

Gopalswamy, N., Hanaoka, Y., Kundu, M. R. et al.: 1997, Astrophys. J., 475, 348, 1997.

Gopalswamy, N., Nitta, N., Manoharan, P. K., Raoult, A., and Pick, M.: 1999, Astron. Astrophys., 347, 684

Hanaoka, Y. and Shinkawa, T.: 1999, Astrophys. J., 510, 466.

Innes, D. E., Inhester, B., Axford, W. I. and Wilhelm, K.: 1997, Nature, 386, 811

Karpen, J. T., Antiochos, S. K. and DeVore, C. R.: 1996, Astrophys. J. Lett., 460, L73

Kosovichev, A. G. and Zharkova, V. V.: 1998, Nature, 393, 317

Koutchmy, S., Hara, H., Suematsu, and Y., Reardon, K.: 1997, Astron. Astrophys., 320, L33-L36 
Krucker, S., Benz, A.: 1998, Astrophys. J., 501, L213

Krucker, S., Benz, A. O., Acton, L. W. and Bastian, T. S.: 1997, Astrophys. J., 488, 499

Kucera, T. A., Aulanier, G., Schmieder, B., Mein, N., and Vial, J.-C.: 1999, Solar Phys., 186,259

Kundu, M. R., Nindos, A., Raulin, J.-P., et al.: 1999, Astrophys. J. , in press

Lean, J.: 1997, Annu. Rev. Astron. Astrophys., 35, 33

Lites, B. W., Martinez-Pillet, V. and Skumanich, A.: 1994, Solar Phys., 155, 11

Lites, B. W., Thomas, J. H., Bogdan, T. J., and Cally, P. S.: 1998, Astrophys. J., 497,464

Lockwood, M, Stamper, R., and Wild, M.N.: 1999, Nature, 399, 437

Low, B. C.: 1996, Solar Phys., 167, 217

Montesinos, B., Thomas, J. H.: 1997, Nature, 390, 485

Moses, J. D., Clette, F., Delaboudiniere, J.-P. et al.: 1997, Solar Phys., 175, 571.

Nindos, A., Kundu, M. R., and White, S. M.: 1999, Astrophys. J., in press

Ohyama, M. and Shibata, K. 1997, Pub. Astron. Soc. Japan, 49, 249-261.

Ohyama, M. and Shibata, K. 1998, Astrophys. J. 499, 934

Parker, E.N.: 1999, Nature, 399, 416

Pevtsov, A. A., Canfield, R. C., and Metcalf, T. R.: 1995, Astrophys. J., 440, L109

Pick, M. :1999, in Solar Physics with Radio Observations, edited by T. Bastian, N. Gopalswamy and K. Shibasaki, in press

Plaza, C. W., Iniesta, J. C. T., Cobo, R. B.,Oillet, V. M., Lites, B. and Skumanich, A.: 1997, Nature, 389, 47

Priest, E. R. and Titov, V.: 1996, Phil. Trans. R. Soc. Lond. A, 354, 2951

Rayrole, J., Mein, P., and Schmieder, B.: 1998, Second Advances in Solar Physics Euroconferences: Three-Dimensional Structure of Solar Active Regions, ASP Conf. Series 155, Ed. by C. E. Alissandrakis and B. Schmieder, ISBN 1-886733-75-9, p260

Schlichenmaier, R., Jahn, K. and Schmidt, H. U.: 1998, Astron. Astrophys., 337, 897

Schmieder, B., Aulanier, G., Démoulin, P. et al.: 1997, Astron. Astrophys., 325, 1213

Schrijver, C. J., A. M. Title, Harvey, K. L., Sheeley Jr, N. R., Wang, Y.-M, van den Oord, G.H.J., Shine, R. A., Tarbell, T. D. and Hurburt, N. E.: 1998, Nature, 394, 439

Shibata, K.: 1996, Adv. Space Res., 17(4/5), 9

Sterling, A. C. and Hudson, H. S.: Astrophys. J., 491, L55

Subramanian, P., Dere, K.P., and Howard, R.A.: 1999, Astrophys. J., in press

Svensmark, H. and Friis-Christensen, E.: 1997, J. Atmos. Sol. Terr. Phys., 59, 1225

Thomas, J. H.: 1998, Nature, 396, 1998, 114

Tsuneta, S. and Naito, T.: 1998, Astrophys. J., 495, L67

Wang, H., Denker, C., Spirock, T., Goode, P. R. et al.: 1998, Solar Phys., 183, 1

Wang, J-X, Li, W., Denker, C. et al.: 1999, BBSO preprint

Wang, T-J, Wang H.-N., and Qiu, J.: 1999, Astron. Astrophys., 342,854

Wang, Y.-M., Sheeley, Jr., N. R., Socker, G. E. et al.: 1998, Astrophys. J., 508, 899.

Zirker, J. B., Martin, S. F., Harvey, K. and Gaizauskas, V.: 1997, Solar Phys., 175, 27 